\title{
A NOTE ON FAMILIES OF HYPERELLIPTIC CURVES
}

\author{
SERGEY GORCHINSKIY AND FILIPPO VIVIANI
}

\begin{abstract}
We give a stack-theoretic proof for some results on families of
\end{abstract} hyperelliptic curves.

\section{INTRODUCTION}

Let $k$ be a field and $g$ be an integer such that $\operatorname{char}(k) \neq 2$ and $g \geq 2$. All schemes that we consider are of finite type over $k$.

Any family $\mathcal{F} \rightarrow S$ of smooth genus $g$ hyperelliptic curves is a double cover of a conic bundle $\mathcal{C} \rightarrow S$ branched at a Cartier divisor $D$ finite and étale of degree $2 g+2$ over the base $S$ (see [LK79]). Conversely, starting with a family $(\mathcal{C} \rightarrow S, D)$ as above, one can ask what are the obstructions to the existence of a corresponding family of hyperelliptic curves $\mathcal{F} \rightarrow S$ and how many such families does there exist. The classical theory of double covers immediately gives the answer to this question in terms of the functions on $\mathcal{C}$ and its Picard group $\operatorname{Pic}(\mathcal{C})$.

In Theorem 3.1 we give a different answer to these questions in terms of the geometry of the base $S$. Our proof is completely stack-theoretic and uses the fact that the stack $\mathcal{H}_{g}$ of hyperelliptic curves is a $\mu_{2}$-gerbe over the stack $\mathcal{D}_{2 g+2}$ of conic bundles endowed with an effective Cartier divisor finite and étale of degree $2 g+2$, and the fact that both these stacks have an explicit description as quotient stacks (see AV04 and GV08).

As an application of the Theorem 3.1 we give a proof of two classical facts on families of hyperelliptic curves.

In Proposition 4.7 we prove that there exists a tautological family of hyperelliptic curves over a non-empty open subset of the coarse moduli space $H_{g}$ if and only if $g$ is odd. Moreover, we give a different proof of GV08, Thm. 3.12], stating that such a family never exists over the open subset $H_{g}^{0}$ corresponding to curves without extra-automorphisms apart from the hyperelliptic involution (this is in contrast with the fact that a tautological family exists over the open subset $M_{g}^{0} \subset M_{g}$ of general curves of genus $g \geq 3$ without automorphisms). From this result and the rationality of $H_{g}$ (see Bog86 and Kat84), we deduce that the stack $\mathcal{H}_{g}$ is rational if and only if $g$ is odd (Corollary 4.9).

In Proposition 4.11, we give a different (and for us simpler) proof of a result of Mestrano-Ramanan ([MR85]), stating that a global $g_{2}^{1}$ for a family of hyperelliptic curves exists only in the case $g$ even.

Date: 19 September 2008.

1991 Mathematics Subject Classification. Primary 14H10, 14A20; Secondary 14D22, 14 H45.

Key words and phrases. hyperelliptic curves, stack, families.

The first author was partially supported by the grants RFBR 05-01-00455, Nsh-1987.2008.1, and INTAS no. 05-1000008-8118.

During the preparation of this paper, the second author was supported by a grant from MittagLeffler Institute in Stockholm. 


\section{Notations}

By $\mathcal{H}_{g}, \mathcal{D}_{2 g+2}$, and $H_{g}$ denote the stack of families of genus $g$ smooth hyperelliptic curves, the stack of conic bundles together with an effective Cartier divisor finite and étale of degree $2 g+2$ over the base, and the common coarse moduli space of two stacks above, respectively.

Recall that given a $k$-scheme $X$ and a $k$-group scheme $G$ acting on $X$, the quotient stack, denoted as $[X / G]$, is the category fibered in groupoids over the category of $k$-schemes, whose fiber over a $k$-scheme $S$ is the groupoid whose objects are $G$-torsors $E \rightarrow S$ endowed with a $G$-equivariant morphism $E \rightarrow X$ and whose arrows are isomorphisms of the above objects. In the particular case where $X=$ $\operatorname{Spec}(k)$, we get the classifying stack of $G$, denoted with $B G$, whose fiber over $S$ is the groupoid of $G$-torsors $E \rightarrow S$.

The stacks $\mathcal{H}_{g}$ and $\mathcal{D}_{2 g+2}$ admit the following description as quotient stacks (see [AV04, Cor. 4.7] and [GV08, Prop. 3.4]):

$$
\left\{\begin{array}{l}
\mathcal{H}_{g}=\left[\mathbb{A}_{s m}(2,2 g+2) /\left(G L_{2} / \mu_{g+1}\right)\right] \\
\mathcal{D}_{2 g+2}=\left[\mathbb{A}_{s m}(2,2 g+2) /\left(G L_{2} / \mu_{2 g+2}\right)\right]=\left[\mathbb{B}_{s m}(2,2 g+2) / P G L_{2}\right] \\
H_{g}=\mathbb{B}_{s m}(2,2 g+2) / P G L_{2},
\end{array}\right.
$$

where $\mathbb{A}_{s m}(2,2 g+2)$ is the linear space of degree $2 g+2$ binary forms without multiple roots, $\mathbb{B}_{s m}(2,2 g+2)$ is the projectivization of $\mathbb{A}_{s m}(2,2 g+2)$, and $G L_{2}$ acts on $\mathbb{A}_{s m}(2,2 g+2)$ by the formula $A \cdot f(x)=f\left(A^{-1} \cdot x\right)$.

We briefly recall the notion of the rigidification of a stack (see ACV03, Section $5.1]$ ). Let $\mathcal{X}$ be an algebraic stack over $k$ (even though everything can be extended to a general base scheme), $H$ a commutative $k$-group scheme and assume that for every object $\xi \in \mathcal{X}(T)$ there is an embedding $H_{T} \subset \operatorname{Aut}_{T}(\xi)$ compatible with pullbacks. Then there is an algebraic stack $\mathcal{X}^{H}$ (called the rigidification of $\mathcal{X}$ along $H$ ) together with a smooth morphism of algebraic stacks $\phi: \mathcal{X} \rightarrow \mathcal{X}^{H}$ uniquely determined by the properties:

(i) For any object $\xi \in \mathcal{X}(T)$ with image $\eta:=\phi(\xi) \in \mathcal{X}^{H}(T)$, we have that $H(T)$ lies in the kernel of $\operatorname{Aut}_{T}(\xi) \rightarrow \operatorname{Aut}_{T}(\eta)$.

(ii) The morphism $\mathcal{X} \rightarrow \mathcal{X}^{H}$ is universal for morphisms of stacks $\mathcal{X} \rightarrow \mathcal{Y}$ satisfying (i) above.

Moreover, a moduli space for $\mathcal{X}$ is also a moduli space for $\mathcal{X}^{H}$ and $\mathcal{X}$ is a $H$-gerbe over $\mathcal{X}$, which means that (see Gir71] or [LMB00])

(a) The structure morphism $\phi: \mathcal{X} \rightarrow \mathcal{X}^{H}$ is surjective.

(b) The diagonal $\Delta: \mathcal{X} \rightarrow \mathcal{X} \times{ }_{\mathcal{X}^{H}} \mathcal{X}$ is surjective.

Let $\Psi: \mathcal{H}_{g} \rightarrow \mathcal{D}_{2 g+2}$ be the morphism of stacks sending a family $\mathcal{F} \rightarrow S$ of smooth genus $g$ hyperelliptic curves over $S$ into the underlying conic bundle $\mathcal{C} \rightarrow S$ together with its relative Cartier branch divisor $D \subset \mathcal{C}$. By the above explicit description, it follows that $\Psi: \mathcal{H}_{g} \rightarrow \mathcal{D}_{2 g+2}$ realizes the stack $\mathcal{D}_{2 g+2}$ as the $\mu_{2^{-}}$ rigidification of the stack $\mathcal{H}_{g}$ along the hyperelliptic involution acting on families of hyperelliptic curves. Thus $\mathcal{H}_{g}$ is a $\mu_{2}$-gerbe over $\mathcal{D}_{2 g+2}$ and they have the same coarse moduli space $H_{g}$.

Let $H_{g}^{0} \subset H_{g}$ be the open subset corresponding to hyperelliptic curves without extra-automorphisms apart from the hyperelliptic involution. The preimage $\mathbb{B}_{s m}(2,2 g+2)^{0}$ of $H_{g}^{0}$ in $\mathbb{B}_{s m}(2,2 g+2)$ is exactly the locus where the action of $P G L_{2}$ is free, hence we have $\left[\mathbb{B}_{s m}(2,2 g+2)^{0} / P G L_{2}\right]=: \mathcal{D}_{2 g+2}^{0} \cong H_{g}^{0}$.

For a small category $\mathcal{E}$, by $|\mathcal{E}|$ denote the set of isomorphism classes of objects in $\mathcal{E}$. 


\section{Main statement}

This section is devoted to the proof of the following

Theorem 3.1. For a scheme $S$, consider a family $(\mathcal{C} \stackrel{p}{\longrightarrow} S, D) \in O b\left(\mathcal{D}_{2 g+2}(S)\right)$; denote by $\Psi^{-1}(\mathcal{C} \stackrel{p}{\longrightarrow} S, D)$ the preimage in $\left|\mathcal{H}_{g}(S)\right|$ of the class of $(\mathcal{C} \stackrel{p}{\longrightarrow} S, D)$ in the set $\left|\mathcal{D}_{2 g+2}(S)\right|$. We have:

(i) If $g$ is odd, then the set $\Psi^{-1}(\mathcal{C} \stackrel{p}{\longrightarrow} S, D)$ is non-empty if and only if $p_{*}\left(\omega_{\mathcal{C} / S}^{g+1}(D)\right)$ is 2-divisible in $\operatorname{Pic}(S)$. If $g$ is even, then the set $\Psi^{-1}(\mathcal{C} \stackrel{p}{\longrightarrow}$ $S, D)$ is non-empty if and only if $p_{*}\left(\omega_{\mathcal{C} / S}^{g+1}(D)\right)$ is 2-divisible in $\operatorname{Pic}(S)$, the family $p: \mathcal{C} \rightarrow S$ is the projectivization of a rank two vector bundle $V \rightarrow S$, and $\operatorname{det} V=p_{*}\left(\omega_{\mathcal{C} / S}^{-1}(-2)\right)$ is 2-divisible in $\operatorname{Pic}(S)$.

(ii) If the set $\Psi^{-1}(\mathcal{C} \stackrel{p}{\longrightarrow} S, D)$ is non-empty, then it is a homogeneous space for the group $H_{e t}^{1}\left(S, \mu_{2}\right)$ with respect to the following action: an element of $H_{\text {ét }}^{1}\left(S, \mu_{2}\right)$, corresponding to a double étale cover $\widetilde{S} \rightarrow S$, sends the hyperelliptic family $(\mathcal{F} \rightarrow S)$ to the family $\left(\left(\mathcal{F} \times{ }_{S} \widetilde{S}\right) /(i \times j) \rightarrow \widetilde{S} / j=S\right)$, where $i$ is the hyperelliptic involution of $\mathcal{F}$ and $j$ is the non-trivial automorphism of $\widetilde{S}$ over $S$. Moreover, if $(\mathcal{C} \stackrel{p}{\longrightarrow} S, D) \in O b\left(\mathcal{D}_{2 g+2}^{0}(S)\right)$, then the action of $H_{\text {ét }}^{1}\left(S, \mu_{2}\right)$ on $\Psi^{-1}(\mathcal{C} \stackrel{p}{\longrightarrow} S, D)$ is free.

The proof of the theorem uses a general result on quotient stacks. Let $G$ be a smooth group scheme acting on a scheme $X$. The natural morphism of quotient stacks $[X / G] \rightarrow B G=[\operatorname{Spec}(k) / G]$ induces a map of sets $|[X / G](S)| \rightarrow|B G(S)|=$ $H_{e t}^{1}(S, G)$ for any scheme $S$, where we used the fact that, since $G$ is a smooth group scheme, the isomorphism classes of $G$-torsor over $S$ are parametrized by the first étale cohomology group of $S$ with coefficients in $G$ (see [MIL80] or Gir71]).

Suppose that we are given a central extension of smooth group schemes

$$
1 \rightarrow K \rightarrow G \rightarrow H \rightarrow 1
$$

and an action of $H$ on a scheme $X$. Consider the "restriction" of this action to $G$. Then for each scheme $S$ the group $H_{e t}^{1}(S, K)$ acts naturally on the set $|[X / G](S)|$ by the formula $\left\{U_{\alpha}, f_{\alpha}, g_{\alpha \beta}\right\} \mapsto\left\{U_{\alpha}, f_{\alpha}, g_{\alpha \beta} k_{\alpha \beta}\right\}$, where $\left\{U_{\alpha}\right\}$ is an étale covering of $S$, the collection $\left\{f_{\alpha}: U_{\alpha} \rightarrow X, g_{\alpha \beta}: U_{\alpha} \times_{S} U_{\beta} \rightarrow G\right\}$ represents an element from $|[X / G](S)|$, and a 1-cocycle $\left\{k_{\alpha \beta}\right\}$ represents an element from $H_{e ́ t}^{1}(S, K)$.

\section{Lemma 3.2.}

(i) For a scheme $S$, the natural map $|[X / G](S)| \rightarrow|[X / H](S)|$ defines a bijection between $|[X / G](S)| / H_{e ́ t}^{1}(S, K)$ and the preimage of the trivial cohomology class under the composition $|[X / H](S)| \rightarrow H_{\text {ét }}^{1}(S, H) \stackrel{\partial}{\longrightarrow} H_{e ́ t}^{2}(S, K)$.

(ii) If the action of $H$ on $X$ is free, then the action of the group $H_{\text {ét }}^{1}(S, K)$ on the set $|[X / G](S)|$ is free.

Proof. The proof of (i) is a direct check, which uses the exact sequence of pointed sets

$$
1 \rightarrow H_{e ́ t}^{1}(S, G) / H_{e ́ t}^{1}(S, K) \rightarrow H_{e ́ t}^{1}(S, H) \rightarrow H_{e ́ t}^{2}(S, K)
$$

In order to prove (ii), suppose that $\left\{U_{\alpha}, f_{\alpha}, g_{\alpha \beta}\right\}$ and $\left\{k_{\alpha \beta}\right\}$ represent elements from $|[X / G](S)|$ and $H_{e ́ t}^{1}(S, K)$ such that $\left\{U_{\alpha}, f_{\alpha}, g_{\alpha \beta}\right\}$ is equivalent to $\left\{U_{\alpha}, f_{\alpha}\right.$, $\left.g_{\alpha \beta} k_{\alpha \beta}\right\}$ in $|[X / G](S)|$. Then, after passing to a subcovering, we see that there exists a collection $\left\{g_{\alpha}: U_{\alpha} \rightarrow G\right\}$ such that $f_{\alpha}=g_{\alpha} f_{\alpha}$ and $g_{\alpha \beta}=g_{\alpha}^{-1} g_{\alpha \beta} k_{\alpha \beta} g_{\beta}$. If the action of $H$ on $X$ is free, then we have $g_{\alpha}: U_{\alpha} \rightarrow K$ for all $\alpha$ and therefore the class of the cocycle $\left\{k_{\alpha \beta}\right\}$ in $H_{e t}^{1}(S, K)$ is trivial. 
Proof of Theorem 3.1. We use Lemma 3.2 with $X=\mathbb{A}_{s m}(2,2 g+2), K=\mu_{2}$, $G=G L_{2} / \mu_{g+1}, H=G L_{2} / \mu_{2 g+2}$.

Using the explicit description of the stack of hyperelliptic families as a quotient stack (see [AV04, Rmk. 3.3 and Thm. 4.1]), one deduces that the "stack-theoretic" action of $H_{e ́ t}^{1}\left(S, \mu_{2}\right)$ on $\left|\mathcal{H}_{g}(S)\right|$ coincides with the one described in the statement of the theorem. This implies Theorem 3.1(ii).

To prove Theorem 3.1(i), we compute explicitly the obstruction map. Recall that there is an isomorphism of algebraic groups $G L_{2} / \mu_{2 g+2} \cong \mathbb{G}_{m} \times P G L_{2}$, given by the formula $[A] \mapsto\left(\operatorname{det}(A)^{g+1},[A]\right)$. Using [GV08, Prop. 4.6], it is easy to show that the map $\left|\mathcal{D}_{2 g+2}(S)\right| \rightarrow H_{e ́ t}^{1}\left(S, G L_{2} / \mu_{2 g+2}\right)$ sends the class of a family $(\mathcal{C} \stackrel{p}{\longrightarrow}$ $S, D)$ to the pair $\left(p_{*}\left(\omega_{\mathcal{C} / S}^{-g-1}(-D)\right), \mathcal{C} \stackrel{p}{\longrightarrow} S\right) \in H_{e ́ t}^{1}\left(S, G L_{2} / \mu_{2 g+2}\right)=H_{e ́ t}^{1}\left(S, \mathbb{G}_{m}\right) \times$ $H_{e ́ t}^{1}\left(S, P G L_{2}\right)$.

For $g$ odd, the isomorphism $G L_{2} / \mu_{g+1} \cong \mathbb{G}_{m} \times P G L_{2}$, given by $[A] \mapsto\left(\operatorname{det}(A)^{\frac{g+1}{2}}\right.$, $[A])$, shows that the exact sequence $(*)$ coincides with the exact sequence

$$
1 \rightarrow \mu_{2} \rightarrow \mathbb{G}_{m} \times P G L_{2} \stackrel{(2,1)}{\longrightarrow} \mathbb{G}_{m} \times P G L_{2} \rightarrow 1
$$

For $g$ even, the isomorphism $G L_{2} / \mu_{g+1} \cong G L_{2}$, given by $[A] \mapsto \operatorname{det}(A)^{\frac{g}{2}} A$, shows that the exact sequence $(*)$ coincides with the exact sequence

$$
1 \rightarrow \mu_{2} \rightarrow G L_{2} \stackrel{(\operatorname{det},[\cdot])}{\longrightarrow} \mathbb{G}_{m} \times P G L_{2} \rightarrow 1 .
$$

Hence in both cases the composition $\operatorname{Pic}(S)=H_{e ́ t}^{1}\left(S, \mathbb{G}_{m}\right) \rightarrow H_{e ́ t}^{1}\left(S, G L_{2} / \mu_{2 g+2}\right)$ $\rightarrow H^{2}\left(S, \mu_{2}\right)$ is equal to the coboundary map arising from the Kummer exact sequence for $\mu_{2}$. Thus this composition vanishes at $p_{*}\left(\omega_{\mathcal{C} / S}^{-g-1}(-D)\right) \in \operatorname{Pic}(S)$ if and only if $p_{*}\left(\omega_{\mathcal{C} / S}^{-g-1}(-D)\right)$ is 2-divisible in $\operatorname{Pic}(S)$.

Further, for $g$ odd, the composition $H_{e ́ t}^{1}\left(S, P G L_{2}\right) \rightarrow H_{e ́ t}^{1}\left(S, G L_{2} / \mu_{2 g+2}\right) \rightarrow$ $H^{2}\left(S, \mu_{2}\right)$ is trivial. For $g$ even, this composition is equal to the coboundary map arising from the exact sequence

$$
1 \rightarrow \mu_{2} \rightarrow S L_{2} \rightarrow P G L_{2} \rightarrow 1 \text {. }
$$

This coboundary map vanishes at the conic bundle $\mathcal{C} \stackrel{p}{\longrightarrow} S$ if and only if $\mathcal{C}=\mathbb{P}(V)$ for a rank two vector bundle $V \rightarrow S$ such that $\operatorname{det}(V)=p_{*}\left(\omega_{\mathcal{C} / S}^{-1}(-2)\right)$ is 2-divisible in $\operatorname{Pic}(S)$. This concludes the proof of Theorem 3.1 .

\section{EXAMPLES AND APPLICATIONS}

First let us discuss the conditions of Theorem 3.1

Using the 2-divisibility conditions in Theorem 3.1 (i), one can easily deduce the 2-divisibility of the line bundle $\mathcal{O}_{\mathcal{C}}(D)$ in the Picard group $\operatorname{Pic}(\mathcal{C})$. The converse seems not to be quite trivial for $g$ even. More precisely, Theorem 3.1(ii) implies the following result.

Corollary 4.1. Let $\mathbb{P}(V) \rightarrow S$ be the projectivization of a rank two vector bundle $V$ on $S$ and let $D \subset \mathbb{P}(V)$ be an effective Cartier divisor finite and étale over $S$ of degree $d \equiv 2(\bmod 4)$. Suppose that $\mathcal{O}_{\mathbb{P}(V)}(D)$ is 2-divisible in $\operatorname{Pic}(\mathbb{P}(V))$. Then $\operatorname{det}(V)$ must be 2-divisible in $\operatorname{Pic}(S)$.

In Corollary 4.1 the hypothesis $D$ being étale over $S$ is necessary as is shown the following example.

Example 4.2. Consider the Hirzebruch surface $p: \mathbb{F}_{n}=\mathbb{P}\left(\mathcal{O}_{\mathbb{P}^{1}} \oplus \mathcal{O}_{\mathbb{P}^{1}}(-n)\right)=$ $\mathbb{P}(V) \rightarrow \mathbb{P}^{1}=S$ for some $n \geq 0$. By [Har, V.2.18], there exists an irreducible smooth curve $D$ in the linear system $\left|d c_{1}+a f\right|$ if $d, a>0$, where $c_{1}$ denotes the first Chern class of the line bundle $\mathcal{O}_{\mathbb{F}_{n}}(1)$ and $f$ denotes the class of a fiber of $p$. 
Then $\mathcal{O}_{\mathbb{F}_{n}}(D)$ is 2-divisible in $\operatorname{Pic}\left(\mathbb{F}_{n}\right)$ if $d$ and $a$ are even, while $\operatorname{det}(V)=\mathcal{O}_{\mathbb{P}^{1}}(-n)$ is not 2-divisible in $\operatorname{Pic}\left(\mathbb{P}^{1}\right)$ if $n$ is odd. Clearly, $D$ is not étale over $S=\mathbb{P}^{1}$.

In Theorem 3.1(i), all the divisibility conditions are necessary as it is shown by the following two examples.

Example 4.3. Suppose that char $(k)$ does not divide $2 g+2$. Consider a general divisor $F \subset \mathbb{P}^{1} \times \mathbb{P}^{2}$ of bi-degree $(2 g+2,1)$. Let $R \subset \mathbb{P}^{2}$ be the ramification curve of the map $p: F \rightarrow \mathbb{P}^{2}$ induced by the second projection. We put $S=\mathbb{P}^{2} \backslash R$, $\mathcal{C}=\mathbb{P}^{1} \times S, D=\left.F\right|_{S}, V=\mathcal{O}_{S} \oplus \mathcal{O}_{S}$. Then the map $p: D \rightarrow S$ is étale of degree $2 g+2$ and $D \subset \mathcal{C}$ is a Cartier divisor since $\mathcal{C}$ is smooth. Clearly, $\operatorname{det} V=\mathcal{O}_{S}$ is 2-divisible in $\operatorname{Pic}(S)$. Further, $\mathcal{O}_{\mathcal{C}}(D)=\mathcal{O}_{\mathcal{C}}(2 g+2) \otimes p^{*}\left(\mathcal{O}_{S}(1)\right)$. Moreover, $R$ is irreducible and it is easily shown that $R$ has the even degree $4 g+2$, hence $\mathcal{O}_{S}(1)$ is not 2-divisible in $\operatorname{Pic}(S)$. Therefore, $p_{*}\left(\omega_{\mathcal{C} / S}^{g+1}(D)\right)$ is not 2-divisible in $\operatorname{Pic}(S)$ (we use that $\left.\omega_{\mathcal{C} / S}(2)=p^{*}(\operatorname{det} V)^{-1}=\mathcal{O}_{\mathcal{C}}\right)$.

Example 4.4. For simplicity, suppose that $\operatorname{char}(k)=0$. Consider the blow-up $P$ of $\mathbb{P}^{3}$ at a point $x \in \mathbb{P}^{3}$. By $\sigma: P \rightarrow \mathbb{P}^{3}$ and $p: P \rightarrow \mathbb{P}^{2}$ denote the corresponding natural maps and by $E \subset P$ denote the exceptional divisor. Recall that $P \cong \mathbb{P}(V)$, where $V=\mathcal{O}_{\mathbb{P}^{2}} \oplus \mathcal{O}_{\mathbb{P}^{2}}(1)$. Let $T \subset \mathbb{P}^{3}$ be a general surface of degree $2 g+3$ such that $T$ contains $x$. Let $R \subset \mathbb{P}^{2}$ be the ramification curve of the degree $2 g+2$ map $p: F \rightarrow \mathbb{P}^{2}$, where $F=\sigma^{*}(T)-E$. We put $S=\mathbb{P}^{2} \backslash R, \mathcal{C}=\left.P\right|_{S}, D=\left.F\right|_{S}$. Then the map $p: D \rightarrow S$ is étale of degree $2 g+2$ and $D \subset \mathcal{C}$ is a Cartier divisor since $\mathcal{C}$ is smooth. Further, $R$ is irreducible and it is easily shown that $R$ has the even degree $(2 g+3)(2 g+2)-2$, hence $\operatorname{det} V=\mathcal{O}_{S}(1)$ is not 2-divisible in $\operatorname{Pic}(S)$. Moreover, $\mathcal{O}_{\mathcal{C}}(D) \equiv \mathcal{O}_{\mathcal{C}}(2 g+2) \otimes p^{*}\left(\mathcal{O}_{S}(-1)\right) \bmod p^{*}(2 \operatorname{Pic}(S))$, therefore $p_{*}\left(\omega_{\mathcal{C} / S}^{g+1}(D)\right)$ is 2divisible in $\operatorname{Pic}(S)$ if $g$ is even (we use that $\left.\omega_{\mathcal{C} / S}(2)=p^{*}(\operatorname{det} V)^{-1}=p^{*}\left(\mathcal{O}_{S}(-1)\right)\right)$.

In Theorem 3.1(ii) the action of $H_{e ́ t}^{1}\left(S, \mu_{2}\right)$ on hyperelliptic families over $S$ is not free in the presence of extra-automorphisms as is shown by the following example.

Example 4.5. Let $S=\operatorname{Spec}(A)$ with $A=k\left[T, T^{-1}\right]$, let $g$ be even, and let $Q(x) \in$ $k[x]$ be a degree $g+1$ polynomial without multiple roots. We put

$$
P(x)=Q(x) Q(T / x) x^{g+1} \in A[x] .
$$

Consider the family of hyperelliptic curves $\mathcal{F}$ over $S$ whose affine model is given by $\left\{y^{2}=P(x)\right\}$ (the corresponding family $\mathcal{C}$ equals to $\mathbb{P}^{1} \times S$ ). Then the double étale cover $\widetilde{S}=\operatorname{Spec}(A[\sqrt{T}]) \rightarrow S$ sends $\mathcal{F}$ to the family $\mathcal{F}^{\prime}$ whose affine model is given by $\left\{T y^{2}=P(x)\right\}$. The map sending $(x, y)$ to $\left(T / x,\left(y T^{g / 2}\right) / x^{g+1}\right)$ defines an isomorphism between $\mathcal{F}$ and $\mathcal{F}^{\prime}$.

Now we give some applications of Theorem 3.1. First of all, note the following immediate corollary of Theorem 3.1

Corollary 4.6. Let $(\mathcal{C} \rightarrow S, D)$ be as in Theorem [3.1. If $g$ is odd, then for a non-empty open subset $U \subset S$, there exists a hyperelliptic family $\mathcal{F} \rightarrow U$, which corresponds to $\left.\mathcal{C}\right|_{U}$. If $g$ is even, then the above statement is true if and only if $\mathcal{C}$ is Zariski locally trivial, that is there exists a non-empty open subset $V \subset S$ such that $\left.\mathcal{C}\right|_{V} \cong \mathbb{P}^{1} \times V$.

Let us give a solution for the Exercise 2.3 from [HM88] (note that there is a small misprint there: universal should be replaced by tautological) together with a different proof of Theorem 3.12 from GV08.

\section{Proposition 4.7.}

(i) There exists a tautological family of hyperelliptic curves over a non-empty open subset in $H_{g}$ if and only if $g$ is odd. 
(ii) For any $g$, there does not exist a tautological family over $H_{g}^{0}$.

Proof. First we prove (i). Since $H_{g}$ is irreducible, we may replace it by the open subset $H_{g}^{0}$. Further, we have $\mathcal{D}_{2 g+2}^{0} \cong H_{g}^{0}$, hence there exists a universal family $\left(\mathcal{C}_{g} \rightarrow\right.$ $\left.H_{g}^{0}, D_{2 g+2}\right) \in \mathcal{D}_{2 g+2}^{0}\left(H_{g}^{0}\right)$. Explicitly, the universal family $\left(p: \mathcal{C}_{g} \rightarrow H_{g}^{0}, D_{2 g+2}\right)$ is is the $P G L_{2}$-quotient of the family $\left(p_{1}: \mathbb{B}_{s m}(2,2 g+2)^{0} \times \mathbb{P}^{1} \rightarrow \mathbb{B}_{s m}(2,2 g+2)^{0}, \mathbb{D}_{2 g+2}\right)$, where $P G L_{2}$ acts diagonally and $\mathbb{D}_{2 g+2}$ is the tautological divisor. Since the action of $P G L_{2}$ is free, it follows from [GIT, Chapter 1, Section 3] that

$$
\operatorname{Pic}\left(\mathcal{C}_{g} / H_{g}^{0}\right)=\operatorname{Pic}^{P G L_{2}}\left(\mathbb{P}^{1}\right)=\mathbb{Z} \cdot \mathcal{O}_{\mathbb{P}^{1}}(2) .
$$

Thus the conic bundle $\mathcal{C}_{g} \rightarrow H_{g}^{0}$ does not have any line bundle of relative degree 1. Hence it can not be the projectivization of a rank two vector bundle on $H_{g}^{0}$ and, by Theorem 3.1(i), we get the first conclusion.

To prove (ii) we are going to show that $p_{*}\left(\omega_{\mathcal{C}_{g} / H_{g}^{0}}^{g+1}\left(D_{2 g+2}\right)\right)$ is not 2-divisible in $\operatorname{Pic}\left(H_{g}^{0}\right)$ if $g \geq 3$, which gives the second conclusion using again Theorem 3.1(i). The $P G L_{2}$-equivariant classes of the tautological divisor $\mathbb{D}_{2 g+2}$ and the relative dualizing sheaf $\omega_{p_{1}}$ for the trivial family $p_{1}$ are given by

$$
\left\{\begin{array}{l}
\mathcal{O}_{\mathbb{B}_{s m}(2,2 g+2)^{0} \times \mathbb{P}^{1}}\left(\mathbb{D}_{2 g+2}\right)=p_{1}^{*} \mathcal{O}_{\mathbb{B}_{s m}(2,2 g+2)^{0}}(1) \otimes p_{2}^{*} \mathcal{O}_{\mathbb{P}^{1}}(2)^{\otimes(g+1)}, \\
\omega_{p_{1}}=p_{2}^{*} \mathcal{O}_{\mathbb{P}^{1}}(2)^{\otimes-1} .
\end{array}\right.
$$

Using the projection formula, we deduce that $p_{*}\left(\omega_{\mathcal{C}_{g} / H_{g}^{0}}^{g+1}\left(D_{2 g+2}\right)\right)$ is equal to

$$
p_{1 *}\left(\omega_{p_{1}}^{g+1}\left(\mathbb{D}_{2 g+2}\right)\right)=\mathcal{O}_{\mathbb{B}_{s m}(2,2 g+2)^{0}}(1) \in \operatorname{Pic}^{P G L_{2}}\left(\mathbb{B}_{s m}(2,2 g+2)^{0}\right)=\operatorname{Pic}\left(H_{g}^{0}\right),
$$

which is not 2-divisible, since $\operatorname{Pic}\left(H_{g}^{0}\right)$ is a finite cyclic group generated by the above element and has even cardinality for $g \geq 3$ ([GV08, Cor. 3.8]).

Remark 4.8. It is interesting to compare the above result with the ones in Mum65] and Ran91. In Mum65, page 58], one can find an explicit tautological family over $H_{1}^{0}$. In Ran91, it is proved that the moduli space of "framed" hyperelliptic curves (i.e. hyperelliptic curve $C$ plus a fixed double cover $C \rightarrow \mathbb{P}^{1}$ ), does have a tautological family over an open subset but not globally.

Proposition 4.7 can be re-interpreted as a result on the rationality of the moduli stack $\mathcal{H}_{g}$. Following [BH06, Section 4], we say that an irreducible algebraic stack $\mathcal{X}$ is rational if it has an open substack isomorphic to $X \times B G$, where $X$ is a rational variety and $G$ is the generic isotropy group of $\mathcal{X}$.

Corollary 4.9. The stack $\mathcal{H}_{g}$ is rational if and only if $g$ is odd.

Proof. The open substack $\mathcal{H}_{g}^{0} \subset \mathcal{H}_{g}$ is a $\mu_{2}$-gerbe over $H_{g}^{0}$. Since $H_{g}$ (and hence $H_{g}^{0}$ ) is rational (see Bog86, Kat84]), $\mathcal{H}_{g}^{0}$ is rational if and only if it is a neutral gerbe locally in the Zariski topology of $H_{g}^{0}$. This is equivalent to the existence of a tautological family Zariski-locally on $H_{g}^{0}$ and hence we conclude by Proposition 4.7

The other application concerns the existence of a global $g_{2}^{1}$ for a hyperelliptic family $\mathcal{F} \stackrel{\pi}{\longrightarrow} S$, i.e., the existence of a line bundle on $\mathcal{F}$ such that its restriction to any geometric fiber of $\pi$ coincides with the unique line bundle of degree 2 and having two independent global sections. We will use the following criterion for the existence of a global $g_{2}^{1}$.

Lemma 4.10. Let $\pi: \mathcal{F} \rightarrow S$ be a family of hyperelliptic curves which is a double cover of the conic bundle $p: \mathcal{C} \rightarrow S$. Assume $S$ is irreducible with generic point $\eta=\operatorname{Spec}(k(S))$. Consider the following conditions: 
(i) There exists a $g_{2}^{1}$ on $\mathcal{F}$.

(ii) The hyperelliptic curve $\mathcal{F}_{\eta}$ admits a $g_{2}^{1}$ defined over $k(S)$.

(iii) The conic $C_{\eta}$ is isomorphic to $\mathbb{P}_{k(S)}^{1}$.

(iv) $p: \mathcal{C} \rightarrow S$ is the projectivization of a rank two vector bundle on $S$.

Then the following implications hold true: (i) $\Rightarrow$ (ii) $\Leftrightarrow$ (iii) $\Leftarrow$ (iv). Moreover, if $S$ is smooth over $k$, then the above conditions are all equivalent.

Proof. The implications (i) $\Rightarrow$ (ii) and (iii) $\Leftarrow$ (iv) are clear. Let us prove the equivalence (ii) $\Leftrightarrow$ (iii). Call $h$ the map from $\mathcal{F}_{\eta}$ to $C_{\eta}$. Now, if $\mathcal{C}_{\eta} \cong \mathbb{P}_{k(S)}^{1}$ then $h^{*}\left(\mathcal{O}_{\mathbb{P}_{k(S)}^{1}}(1)\right)$ provides the required $g_{2}^{1}$ on $\mathcal{F}_{\eta}$. Conversely, if the $g_{2}^{1}$ of $\mathcal{F}_{\eta}$ is defined over $k(S)$ then $V:=\pi_{*}\left(g_{2}^{1}\right)=H^{0}\left(g_{2}^{1}\right)$ is a vector space over $k(S)$ of dimension 2 and, by construction, $\mathcal{C}_{\eta} \cong \mathbb{P}(V)=\mathbb{P}_{k(S)}^{1}$.

Assume now that $S$ is smooth over $k$. Let us prove the implication (i) $\Leftarrow$ (ii). The hypothesis implies that there is an open subset $U \subset S$ such that $\pi^{-1}(U) \rightarrow U$ admits a $g_{2}^{1}$. Since $S$ and $\pi$ are smooth (and hence also $\mathcal{F}$ ), we can extend the above line bundle to a line bundle, call it $L$, on $\mathcal{F}$ (simply take the closure of the Cartier $=$ Weyl divisor associated to it). The line bundle $L$ has vertical degree 2 everywhere since the vertical degree is locally constant and $S$ is irreducible, and moreover $h^{0}\left(\mathcal{F}_{s}, L_{\mid \mathcal{F}_{s}}\right) \geq 2$ for every geometric point $s$ of $S$, by semicontinuity of $h^{0}$. This implies that $L$ is the required $g_{2}^{1}$ on $\mathcal{F}$.

Let us finally prove the implication (iii) $\Rightarrow$ (iv) assuming that $S$ is smooth. The hypothesis implies that there exists an open subset $U \subset S$ such that $p^{-1}(U) \rightarrow U$ admits a line bundle of vertical degree one. As before, using that $\mathcal{C}$ is smooth (since $p$ and $S$ are smooth), we can extend this line bundle to a line bundle, call it $M$, on $\mathcal{C}$ that will have vertical degree one. Since the geometric fibers of $p$ are $\mathbb{P}^{1}$, we have that $p_{*}(M)$ is a locally free sheaf of rank 2 . The natural map $p^{*}\left(p_{*}(M)\right) \rightarrow$ $M$ is surjective since its restriction to every geometric fiber is surjective. Hence it determines an $S$-map $\Phi: \mathcal{C} \rightarrow \mathbb{P}\left(p_{*}(M)\right)$ that, being an isomorphism on the geometric fibers, is an isomorphism.

Proposition 4.11. If $g$ is odd, then there does not exist a global $g_{2}^{1}$ for any tautological family over a non-empty open subset in $H_{g}$. If $g$ is even, then a global $g_{2}^{1}$ exists for any family of genus $g$ hyperelliptic curves over an irreducible smooth $k$-scheme.

Proof. If $g$ is odd then, from the proof of Proposition 4.7, we known that the universal conic bundle $\mathcal{C}_{g} \rightarrow H_{g}^{0}$ is not Zariski locally trivial and therefore we conclude by the implication (i) $\Rightarrow$ (iii) of the above Lemma 4.10 .

If $g$ is even and $\mathcal{F} \rightarrow S$ is a family of hyperelliptic curves realized as a double cover of the conic bundle $\mathcal{C} \rightarrow S$, then Theorem 3.1(i) gives that $\mathcal{C} \rightarrow S$ is the projectivization of a rank two vector bundle on $S$. If, moreover, $S$ is irreducible and smooth over $k$, then there exists a global $g_{2}^{1}$ on $\mathcal{F}$ by the implication (iv) $\Rightarrow$ (i) of the above Lemma 4.10 .

\section{REFERENCES}

[ACV03] D. Abramovich, A. Corti, A. Vistoli, Twisted bundles and admissible covers. Special issue in honor of Steven L. Kleiman. Comm. Algebra 31 (2003), 3547-3618.

[AV04] A. Arsie, A. Vistoli, Stacks of cyclic covers of projective spaces. Compos. Math. 140 (2004), 647-666.

[BH06] I. Biswas, N. Hoffmann, Some moduli stacks of symplectic bundles on a curve are rational. Adv. Math. 219 (2008), 1150-1176.

[Bog86] F. A. Bogomolov, Rationality of the moduli of hyperelliptic curves of arbitrary genus. Proceedings of the 1984 Vancouver conference in algebraic geometry, 17-37, CMS Conf. Proc., 6, Amer. Math. Soc., Providence, RI, 1986. 
[Gir71] J. Giraud, Cohomologie non abélienne. Die Grundlehren der mathematischen Wissenschaften, Band 179. Springer-Verlag, Berlin-New York, 1971.

[GV08] S. Gorchinskiy, F. Viviani, Picard group of moduli of hyperelliptic curves. Math. Zeit. 258 (2008), 319-331.

[HM88] J. Harris, I. Morrison, Moduli of curves. Graduate Texts in Mathematics, 187. SpringerVerlag, New York, 1998.

[Har] R. Hartshorne, Algebraic geometry. Graduate Texts in Mathematics, 52. Springer-Verlag, New York- Heidelberg, 1977.

[Kat84] P. I. Katsylo, Rationality of the moduli spaces of hyperelliptic curves (Russian). Izv. Akad. Nauk SSSR Ser. Mat. 48 (1984), 705-710.

[LMB00] G. Laumon, L. Moret-Bailly, Champs algébriques. Ergebnisse der Mathematik und ihrer Grenzgebiete 3. Folge, A Series of Modern Surveys in Mathematics 39, Springer-Verlag, Berlin 2000.

[LK79] K. Lonsted, S. L. Kleiman, Basics on families of hyperelliptic curves. Compositio Math. 38 (1979), 83-111.

[MR85] N. Mestrano, S. Ramanan, Poincaré bundles for families of curves. J. Reine Angew. Math. 362 (1985), 169-178.

[MIL80] J. S. Milne, Étale cohomology. Princeton Mathematical Series 33, Princeton University Press, Princeton N.J. 1980.

[GIT] D. Mumford, Geometric invariant theory. Ergebnisse der Mathematik und iher Grenzgebiete, Neue Folge, Band 34 Springer-Verlag, Berlin-New York 1965.

[Mum65] D. Mumford, Picard groups of moduli problems. Arithmetical Algebraic Geometry (Proc. Conf. Purdue Univ., 1963) pp. 33-81, Harper and Row, New York.

[Ran91] Z. Ran, A remark on hyperelliptic curves. Arch. Math. (Basel) 57 (1991), 622-624.

Steklov Mathematical Institute, Gubkina str. 8, 119991 Moscow (Russia)

E-mail address: gorchins@mi.ras.ru

Institut für Mathematik, Humboldt Universität Zu Berlin, 10099 Berlin (Germany). E-mail address: viviani@math.hu-berlin.de 